\title{
Erratum to: Classifying Hilbert functions of fat point subschemes in $\mathbb{P}^{2}$
}

\author{
A. V. Geramita · Brian Harbourne · Juan Migliore
}

Published online: 12 January 2011

(C) Universitat de Barcelona 2011

\section{Erratum to: Collect. Math. DOI 10.1007/BF03191208}

Some of the tables in the originally published version of this paper had errors (see [1]). Corrections have been made to Tables 2, 4 and 7. A version of the paper with corrected tables has been posted on the arXiv as arXiv:0803.4113, version 3 .

The corrections are as follows. Line 2 of Table 2 indicated that the Hilbert function $h_{Z}$ was " 1 " for a fat point subscheme $Z=2 p$, given a point $p \in \mathbb{P}^{2}$, but of course $h_{Z}$ in this case is " 13 ".

The configuration types as listed in Table 4 are not consistent with the types as defined in Table 3. For example, type 1 in Table 4 refers to 7 points on an irreducible conic, but this configuration is defined to be type 25 in Table 3 . Here is a corrected version of Table 4, which is now consistent with the originally published Table 3 .

The online version of the original article can be found under doi:10.1007/BF03191208.

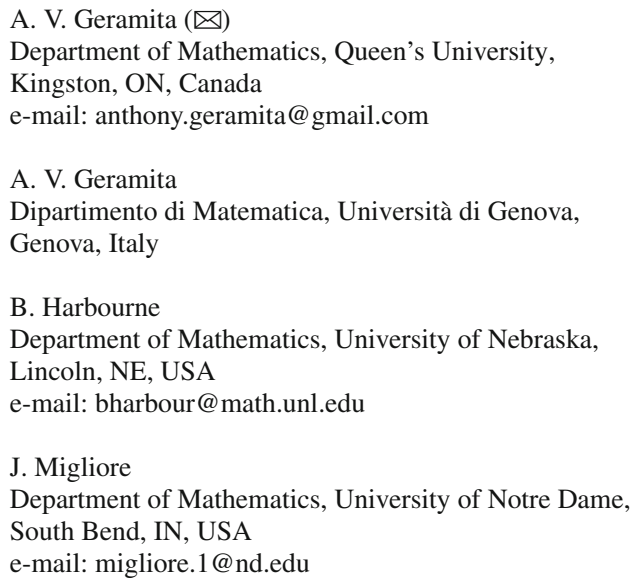


Table 4 Hilbert functions by configuration type for $r=7$ points

\begin{tabular}{llll}
\hline$r$ & $m$ & Type(s) & $h_{Z}$ \\
\hline 7 & 1 & $8,9,25$ & $1,3,5,7$ \\
7 & 2 & 8,25 & $1,3,6,10,14,18,20,21$ \\
7 & 2 & 9 & $1,3,6,10,14,17,19,21$ \\
\hline 7 & 1 & $1,5,6,10,11,12,13, \ldots, 24,26, \ldots, 29$ & $1,3,6,7$ \\
7 & 2 & $11,14,18,21,26,27,28,29$ & $1,3,6,10,15,20,21$ \\
7 & 2 & $5,10,13,17$ & $1,3,6,10,15,19,20,21$ \\
7 & 2 & $1,6,12,15,16,19,20,22,23,24$ & $1,3,6,10,15,21$ \\
\hline 7 & 1 & 2 & $1,2,3,4,5,6,7$ \\
7 & 2 & 2 & $1,3,5,7,9,11,13,15,16,17,18,19,20,21$ \\
\hline 7 & 1 & 3 & $1,3,4,5,6,7$ \\
7 & 2 & 3 & $1,3,6,10,12,14,16,17,18,19,20,21$ \\
\hline 7 & 1 & 4,7 & $1,3,5,6,7$ \\
7 & 2 & 4,7 & $1,3,6,10,14,17,18,19,20,21$ \\
\hline
\end{tabular}

Finally, two changes are being made to Table 7. The fourth line of Table 7 has an unnecessary repeated entry. There is also a missing line, which should have immediately followed the fourth line in Table 7. Thus the fourth line of Table 7,

$$
82111,121,1261,3,6,10,15,20,23,24,24,
$$

should be replaced by the following pair of lines:

$$
\begin{array}{llll}
8 & 2 & 111,121,126 & 1,3,6,10,15,20,23,24 \\
8 & 2 & 116,117,118,119 & 1,3,6,10,15,20,22,24
\end{array}
$$

\section{Reference}

1. Geramita, A.V., Harbourne, B., Migliore, J.: Classifying Hilbert functions of fat point subschemes in $\mathbb{P}^{2}$. Collect. Math. 60(2), 159-192 (2009) 